\title{
Association between Nonalcoholic Liver Disease and Chronic Kidney Disease: An Ultrasound Analysis from NHANES 1988-1994
}

\author{
Jeffrey C. Sirota ${ }^{\mathrm{a}}$ Kim McFann ${ }^{\mathrm{a}}$ Giovanni Targher ${ }^{\mathrm{b}}$ Michel Chonchol ${ }^{\mathrm{a}}$ \\ Diana I. Jalal ${ }^{\mathrm{a}}$ \\ a University of Colorado at Denver Health Sciences Center, Aurora, Colo., USA; bSection of Endocrinology and \\ Metabolism, Department of Medicine, University and Azienda Ospedaliera Universitaria Integrata of Verona, \\ Verona, Italy
}

\section{Key Words}

Chronic kidney disease - Nonalcoholic fatty liver disease • NHANES

\begin{abstract}
Background/Aims: Nonalcoholic fatty liver disease (NAFLD) has been proposed to contribute to chronic kidney disease (CKD) independently of traditional cardiometabolic risk factors. We hypothesized that NAFLD is associated with CKD and that greater severity of NAFLD is associated with higher odds of CKD. Methods: A cross-sectional analysis of 11,469 adults who participated in the National Health and Nutrition Examination Survey (NHANES) 1988-1994. NAFLD was defined by ultrasonographic detection of steatosis in the absence of other liver diseases. CKD was defined as an estimated glomerular filtration rate of $\leq 60 \mathrm{ml} / \mathrm{min} / 1.73 \mathrm{~m}^{2}$ or the presence of albuminuria in subjects with an estimated glomerular filtration rate of $>60 \mathrm{ml} / \mathrm{min} / 1.73 \mathrm{~m}^{2}$. Results: 2,891 (25.4\%) patients in the cohort had CKD. The prevalence of NAFLD was higher in individuals with CKD compared to those without CKD (42.2 vs. $34.5 \%$, p < 0.0001). NAFLD was associated with CKD in unadjusted logistic regression analysis $(\mathrm{OR}=1.47,95 \% \mathrm{Cl}: 1.29-1.67, \mathrm{p}<0.0001)$. Adjustment for demographics and components of metabolic syndrome
\end{abstract}

attenuated this relationship (OR $=1.04,95 \% \mathrm{Cl}: 0.88-1.23$, $\mathrm{p}=0.64)$. Moderate and severe NAFLD on ultrasound were increasingly associated with prevalent CKD in unadjusted analysis, but not after adjustment for metabolic syndrome components. Conclusion: After adjusting for features of metabolic syndrome, ultrasound-diagnosed NAFLD is not associated with prevalent CKD among US adults. Aggressive public health efforts are needed to prevent and treat metabolic syndrome.

Copyright $\odot 2012$ S. Karger AG, Basel

\section{Introduction}

Nonalcoholic fatty liver disease (NAFLD) has become increasingly common over the past few decades, with prevalence estimates at 20-30\% in the USA, Europe, and Asia $[1,2]$. Now the most common chronic liver disease in the developed world, its increase in prevalence may be related to its pathogenetic association with the burgeoning epidemics of obesity and metabolic syndrome [3]. This etiologic background has resulted in increasingly recognized associations between NAFLD and other conditions that are pathogenically related to metabolic syndrome, such as cardiovascular disease $[4,5]$.

\section{KARGER}

Fax +41 613061234 E-Mail karger@karger.ch www.karger.com

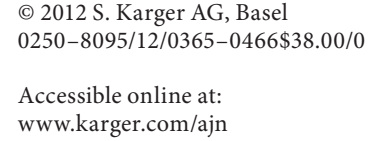

Accessible online at:
www.karger.com/ajn

Diana I. Jalal, MD

12700 East 19th Avenue

Mail Stop C-281

Aurora, CO 80045 (USA)

E-Maildiana.jalal@ucdenver.edu 
Similarly, chronic kidney disease (CKD), is strongly associated with metabolic syndrome and cardiovascular disease [6-8]. In addition to shared risk factors with cardiovascular and kidney disease, it has been proposed that NAFLD itself may contribute to subclinical systemic inflammation and endothelial dysfunction $[9,10]$, thus contributing to kidney and cardiovascular disease. Consistent with this hypothesis, a few observational studies have shown an association of NAFLD with prevalent CKD independently of traditional cardiometabolic risk factors [11-14].

Liver ultrasound, a noninvasive and widely available diagnostic tool, has emerged as a viable alternative to liver biopsy in large population-based studies of NAFLD. The role of ultrasound in the diagnosis of NAFLD has been enhanced by the development of a radiographic scoring system, which has ameliorated previous concerns regarding intra- and interobserver reliability [15]. Liver ultrasound data is newly available from the National Health and Nutrition Examination Survey III (NHANES 19881994). In this study, we hypothesized that NAFLD is associated with prevalent $\mathrm{CKD}$, independently of cardiometabolic risk factors, and that greater severity of NAFLD by ultrasound is associated with higher odds of CKD.

\section{Subjects and Methods}

\section{Study Population}

Our analysis is based upon data from the NHANES program, a 40 -year-old research initiative run by the National Center for Health Statistics. Cross-sectional demographic, socioeconomic, dietary, and medical data were collected through surveys, physical examination, and laboratory testing, with oversampling of African Americans, Hispanics, and those over 60 years of age in order to obtain a representative sample of the US adult population.

The database contained 14,797 participants aged $20-74$ years who had undergone liver ultrasonography. Individuals with ungradable or inadequate imaging, individuals with viral hepatitis $\mathrm{B}$ or $\mathrm{C}$, those who reported excessive alcohol intake ( $\geq 2$ drinks/ day), and those with incomplete laboratory or clinical data were excluded from analysis. As a result, 11,469 subjects met our criteria and were included in the final analysis (fig. 1).

\section{Predictors and Outcome Measure}

The independent variable was the presence of NAFLD, as detected by ultrasound. As part of the NHANES program, ultrasonography had been performed through longitudinal and transverse scanning of the right upper quadrant with the patient in both the supine and left lateral decubitus positions. In 2009-2010, trained ultrasound readers examined video recordings of these ultrasounds, specifically evaluating the liver imaging for evidence of hepatic steatosis based on standardized criteria.

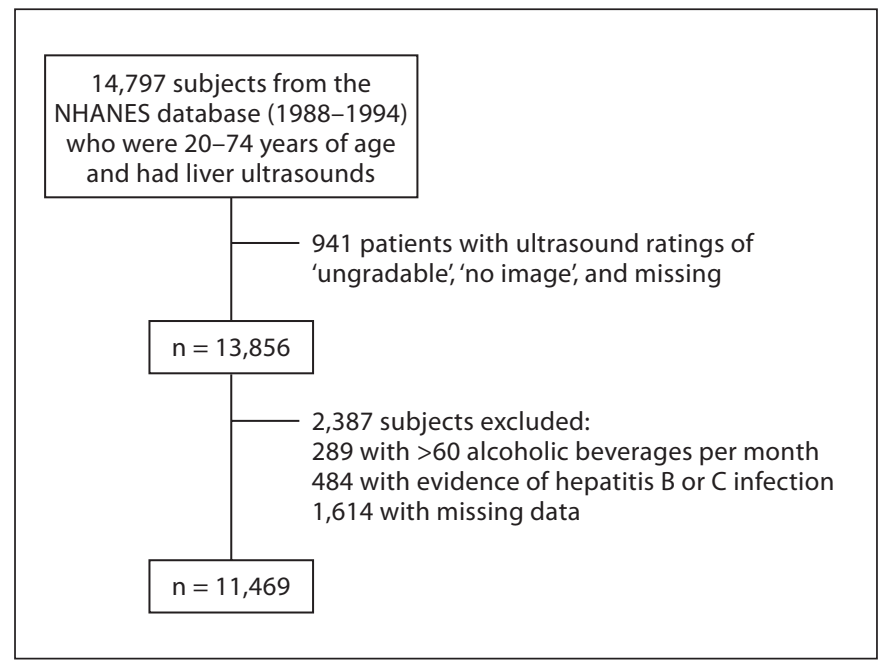

Fig. 1. Study population. Of the initial 14,797 eligible candidates for the study, a total of 11,469 subjects met the inclusion criteria and were included in the analysis.

Briefly, criteria for the diagnosis and characterization of NAFLD include hepatorenal echo contrast, liver parenchymal brightness, deep beam attenuation, and vessel blurring [15]. Hepatorenal echo contrast and liver brightness were considered together and assigned a combined score on a $0-3$ scale. Deep attenuation was scored from $0-2$, and vessel blurring was scored $0-1$. These three separate scores were totaled and, using an algorithm, transformed into an overall NAFLD score that ranged from $1-4$, in which $1=$ absent, $2=$ mild, $3=$ moderate, and $4=$ severe [16].

The dependent variable of interest was the presence of CKD, defined as either an estimated glomerular filtration rate (eGFR) of $\leq 60 \mathrm{ml} / \mathrm{min} / 1.73 \mathrm{~m}^{2}$ or the presence of albuminuria. The eGFR calculation was based on the abbreviated Modification of Diet in Renal Disease study formula: $\mathrm{eGFR}=186.3 \times($ serum creatinine in $\mathrm{mg} / \mathrm{dl})^{-1.154} \times$ age $^{-0.203} \times(0.742$ if female $) \times(1.21$ if black). Albuminuria was defined as a spot urinary albumin-tocreatinine ratio that exceeded $30 \mathrm{mg} / \mathrm{g}$.

\section{Other Covariates}

Covariates were chosen based on prior studies and their biological plausibility. The following covariates were included in the logistic regression analysis: age, sex, race, history of hypertension, history of diabetes, systolic blood pressure (BP), waist circumference, triglycerides, high-density lipoprotein (HDL) cholesterol, and the homeostasis model assessment of insulin resistance (HOMA-IR).

History of hypertension was defined as a physician diagnosis of hypertension or the use of antihypertensive medications. History of diabetes was defined as a physician diagnosis of diabetes or the use of diabetic medications. Systolic BP was measured by physicians at mobile examination centers with mercury sphygmomanometers using a standard protocol. Waist circumference was measured according to NHANES manual of procedures, in which 
tape is applied above the upper border of the right ilium horizontally and then tightened slightly to measure the waist circumference in centimeters (to the nearest millimeter) [16]. Fasting levels of triglycerides and HDL cholesterol were measured enzymatically with a Hitachi 704 Analyzer (Boehringer Mannheim Diagnostics, Indianapolis, Ind., USA). HOMA-IR score was calculated using the HOMA-IR formula [HOMA-IR = fasting insulin $(\mathrm{mU} / \mathrm{l}) \times$ fasting glucose $(\mathrm{mmol} / \mathrm{l}) / 22.5$; the fasting glucose used in this equation was measured by the hexokinase method, and fasting insulin was measured using the Merocodia Insulin ELISA].

\section{Statistical Analysis}

Due to the complex sample strategy of NHANES 1988-1994, appropriate 6-year weights and strata were applied. SAS (9.2) PROC SURVEYMEANS and SURVEYFREQ were used to obtain descriptive statistics for the population. Analysis was performed using the Rao-Scott $\chi^{2}$ test for categorical variables and one-way ANOVA for continuous variables. Logistic regression analysis was applied to examine whether the presence of NAFLD was associated with prevalent CKD. Results are expressed as OR with 95\% CI. Three separate logistic regression models were applied: an unadjusted model; a model adjusted for age, sex, and race (model 1); and a model adjusted for history of hypertension, history of diabetes, systolic BP, waist circumference, triglycerides, HDL cholesterol, and HOMA-IR score (model 2). Logistic regression modeling was also done to examine any association between CKD and the ultrasonographic severity of NAFLD (mild, moderate, and severe). Because diabetes is a well-established and independent risk factor for both NAFLD and CKD, we stratified by diabetes status. Since only $62 \%$ of all participants fasted appropriately prior to measurement of triglycerides and insulin levels, we conducted a sensitivity analysis excluding triglycerides and HOMA-IR from the model and then excluding nonfasting individuals from the full multivariate model (model 2). All statistical analyses were performed using SAS 9.2 software.

\section{Results}

\section{Clinical Characteristics of Participants}

Of the 11,469 participants, 2,891 (25.4\%) had CKD and 8,578 (74.5\%) did not. Clinical characteristics of the study participants stratified by CKD status are shown in table 1. Compared to individuals without CKD, those with CKD were older; more likely to be female and white; and had higher values of waist circumference, systolic BP, plasma triglycerides and HOMA-IR score, and lower HDL cholesterol levels. Subjects with CKD were also far more likely to have a history of hypertension and diabetes than those without CKD.

\section{Relationship between Ultrasound-Diagnosed NAFLD and Prevalent CKD}

A higher percentage of patients with CKD were found to have NAFLD on ultrasound when compared to those
Table 1. Demographic and clinical characteristics of the study population, grouped by CKD status

\begin{tabular}{lccc}
\hline Characteristic & $\begin{array}{l}\text { No CKD } \\
(\mathrm{n}=8,578)\end{array}$ & $\begin{array}{l}\text { CKD } \\
(\mathrm{n}=2,891)\end{array}$ & $\begin{array}{l}\mathrm{p} \\
\text { value }\end{array}$ \\
\hline Age, years & $38 \pm 0.20$ & $53 \pm 0.38$ & $<0.0001$ \\
Male, \% & 48.0 & 37.1 & $<0.0001$ \\
Race, \% & & & \\
$\quad$ White & 64.7 & 75.4 & $<0.0001$ \\
$\quad 31.4$ & 21.6 & \\
$\quad$ Mlack & 0.05 & 0.03 & \\
$\quad$ Othexican-American & 3.9 & 3.0 & \\
History of hypertension, \% & 19.1 & 41.8 & $<0.0001$ \\
History of diabetes, \% & 4.8 & 15.2 & $<0.0001$ \\
Systolic BP, mm Hg & $118 \pm 0.2$ & $129 \pm 0.5$ & $<0.0001$ \\
HDL cholesterol, mg/dl & $50 \pm 0.3$ & $51 \pm 0.4$ & 0.0161 \\
Triglycerides, mg/dl & $134 \pm 2.0$ & $164 \pm 3.1$ & $<0.0001$ \\
Waist circumference, cm & $91 \pm 0.2$ & $95 \pm 0.4$ & $<0.0001$ \\
HOMA-IR score & $2.5 \pm 0.1$ & $3.9 \pm 0.2$ & $<0.0001$ \\
NAFLD prevalence, \% & & & \\
$\quad$ Any NAFLD & 34.5 & 42.2 & $<0.0001$ \\
$\quad$ Mild NAFLD & 13.6 & 13.3 & \\
$\quad$ Moderate NAFLD & 14.2 & 19.4 & \\
$\quad$ Severe NAFLD & 6.8 & 9.4 & \\
\hline
\end{tabular}

Table 2. Logistic regression of CKD on prevalent NAFLD as detected by ultrasound in the whole cohort of NHANES participants

\begin{tabular}{lccc}
\hline Regression models & OR & $95 \%$ CI & $\begin{array}{l}\mathrm{p} \\
\text { value }\end{array}$ \\
\hline Unadjusted model & 1.47 & $1.29-1.67$ & $<0.0001$ \\
\hline $\begin{array}{l}\text { Model 1 } \\
\text { Adjusted for age, race, and sex }\end{array}$ & 1.21 & $1.04-1.40$ & 0.014 \\
\hline $\begin{array}{l}\text { Model 2 } \\
\begin{array}{l}\text { Adjusted for age, race, sex, history of } \\
\text { hypertension or diabetes, systolic BP, } \\
\text { waist circumference, triglycerides, }\end{array}\end{array}$ & 1.04 & $0.88-1.23$ & 0.64 \\
\begin{tabular}{l} 
HDL cholesterol, and HOMA-IR \\
\hline
\end{tabular} & & & \\
\hline
\end{tabular}

without CKD (42.2 vs. $34.5 \%$; $\mathrm{p}<0.0001$ ). As shown in table 1, this increased overall prevalence was mainly due to the higher prevalence of moderate and severe NAFLD in the CKD population, as both groups had similar rates of mild NAFLD on ultrasound (13.3 vs. $13.6 \%$ in the CKD and non-CKD groups, respectively).

As shown in table 2, logistic regression analysis using an unadjusted model determined that the OR for the presence of CKD among patients with NAFLD was 1.47 


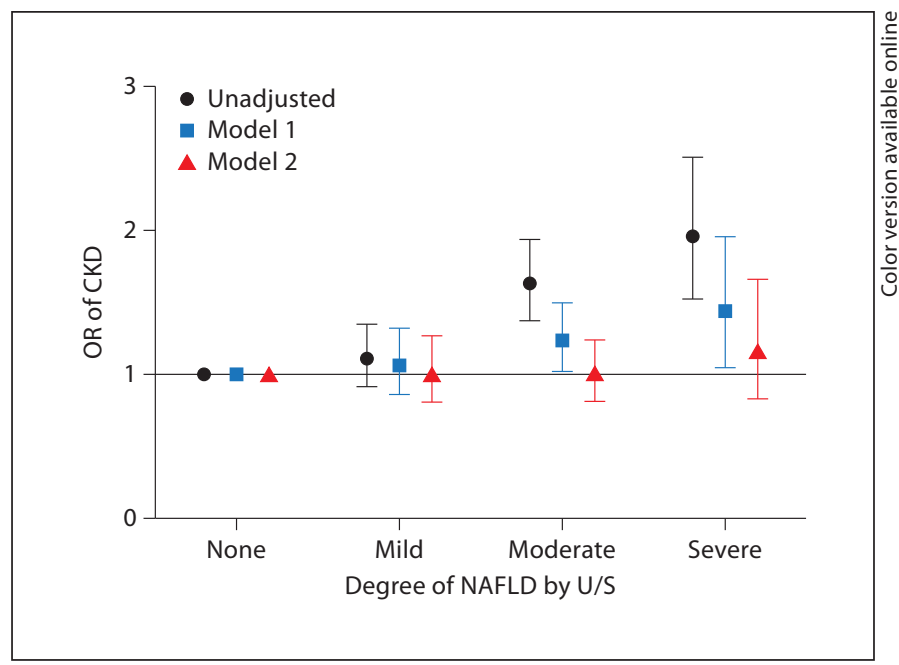

Fig. 2. Odds of prevalent CKD in patients with different ultrasonographic severity (U/S) of NAFLD as compared to patients without NAFLD. In unadjusted analysis, moderate and severe NAFLD were associated with a statistically significant increase in the OR for CKD. This remained significant after adjusting for age, sex, and race, but was attenuated after further adjustment for metabolic syndrome components.

(95\% CI: $1.29-1.67, \mathrm{p}<0.0001)$ when compared to patients without NAFLD. After adjusting for age, sex, and race (model 1), subjects with NAFLD remained at increased odds of prevalent CKD (OR $=1.21,95 \%$ CI: $1.04-$ $1.40, \mathrm{p}=0.014)$. Further adjustment for cardiometabolic risk factors (model 2) attenuated this relationship (OR = 1.04, 95\% CI: 0.88-1.23, $\mathrm{p}=0.64$ ).

\section{Relationship between NAFLD Severity and \\ Prevalent CKD}

Individuals with severe NAFLD by ultrasound were found to have an unadjusted OR for prevalent CKD of 1.96 (95\% CI: 1.53-2.51, $\mathrm{p}<0.001$ ) when compared to patients without NAFLD. Adjustment for age, race, and sex (model 1) yielded an OR of 1.44 (95\% CI: 1.05-1.96, p = 0.02). However, after adjusting for the cardiometabolic risk factors in model 2, the OR of CKD was 1.17 (95\% CI: $0.83-1.65, \mathrm{p}=0.38$ ). Similar to severe NAFLD, moderate NAFLD by ultrasound was associated with higher odds of CKD in unadjusted analysis (OR $=1.64,95 \%$ CI: 1.38 $1.94, \mathrm{p}<0.0001)$. This relation remained significant after adjusting for demographics (OR $=1.24,95 \%$ CI: 1.03 $1.50, \mathrm{p}=0.03)$, but was no longer significant after additional adjustment for cardiometabolic risk factors $(\mathrm{OR}=$ 1.01, 95\% CI: $0.82-1.24, \mathrm{p}=0.92)$. There was no associa- tion between mild NAFLD by ultrasound and CKD in unadjusted or adjusted logistic regression analysis (fig. 2).

\section{Association between NAFLD and CKD by Diabetes Status}

There was no association between NAFLD and CKD in individuals with diabetes in either unadjusted or adjusted regression analyses. Conversely, in subjects without diabetes, the OR for CKD in NAFLD was 1.40 (95\% CI: $1.22-1.61, \mathrm{p}<0.0001)$ in unadjusted analysis, and 1.20 (95\% CI: $1.03-1.41, \mathrm{p}=0.022)$ after adjusting for demographics. The association between NAFLD and CKD was attenuated, however, after further adjustment for the covariates included in model 2 (table 3).

Of note, the association between NAFLD and CKD remains not significant in the whole population and in the participants with and without diabetes mellitus if triglycerides and HOMA-IR are removed from model 2. Similarly, if the nonfasting individuals are excluded from the analysis, there is no significant association between NAFLD and CKD in the fully adjusted model.

\section{Discussion}

This analysis represents the first inquiry into whether an association between ultrasound-diagnosed NAFLD and CKD exists in a large, nationally representative sample of the US population. Our data demonstrate a significant, positive association between NAFLD and prevalent CKD in univariate analysis. This relationship persisted after adjustment for demographics, but was attenuated by adjusting for features of metabolic syndrome. In addition, we report that increasing severity of NAFLD on ultrasound was significantly associated with a greater age, sex, and race-adjusted prevalence of CKD. The association between the ultrasonographic severity of NAFLD and prevalent CKD was also attenuated after adjustment for features of metabolic syndrome.

It has been proposed that NAFLD itself, independently of obesity and other metabolic disorders, could contribute to the development and progression of CKD. In the setting of visceral obesity, insulin resistance leads to increased peripheral lipolysis and a consequent influx of free fatty acids into the liver [10]. The concurrent chronic hyperinsulinemia induces intrahepatic triglyceride production [17], and the consequently increased triglyceride content in the liver (i.e. pure steatosis) can progress to nonalcoholic steatohepatitis in the presence of oxidative stress and inflammation [18-20]. These hepatic necroin- 
Table 3. Logistic regression of CKD on prevalent NAFLD, stratified by diabetes status

\begin{tabular}{|c|c|c|c|c|c|c|}
\hline \multirow[t]{2}{*}{ Regression models } & \multicolumn{3}{|c|}{ Subjects without diabetes } & \multicolumn{3}{|c|}{ Subjects with diabetes } \\
\hline & OR & $95 \% \mathrm{CI}$ & $\mathrm{p}$ value & OR & $95 \% \mathrm{CI}$ & $\mathrm{p}$ value \\
\hline Unadjusted model & 1.40 & $1.22-1.61$ & $<0.0001$ & 1.08 & $0.66-1.76$ & 0.75 \\
\hline $\begin{array}{l}\text { Model } 1 \\
\text { Adjusted for age, race, and sex }\end{array}$ & 1.20 & $1.03-1.41$ & 0.022 & 0.89 & $0.52-1.52$ & 0.67 \\
\hline $\begin{array}{l}\text { Model } 2 \\
\text { Adjusted for age, race, sex, history of hypertension } \\
\text { or diabetes, systolic BP, waist circumference, triglycerides, } \\
\text { HDL cholesterol, and HOMA-IR }\end{array}$ & 1.07 & $0.90-1.27$ & 0.47 & 0.58 & $0.32-1.06$ & 0.47 \\
\hline
\end{tabular}

flammatory changes may exacerbate systemic/hepatic insulin resistance and the metabolic syndrome, and have been suggested to contribute to the development and progression of CKD.

In addition, NAFLD, especially in its necroinflammatory form, may release a variety of cytokines and proinflammatory mediators (such as leptin, resistin, interleukin-6, tumor necrosis factor- $\alpha$, and transforming growth factor- $\beta$ ) [21] that may play a role in CKD pathogenesis [22]. Additionally, decreased levels of adiponectin seen in NAFLD may produce pathological changes in the kidney [23]. The possibility of these pathways producing a link between NAFLD and CKD independent of metabolic syndrome components has been also supported by some recent prospective studies that have shown that pre-existing NAFLD can predict new-onset CKD in individuals with [14] and without diabetes [11].

While the cross-sectional nature of our study precludes us from drawing conclusions about causality between the two disorders in question, the fact that controlling for the features of metabolic syndrome attenuated the association between NAFLD and CKD suggests that the relationship between both diseases may be confounded by some common pathophysiological mechanisms and shared cardiometabolic risk factors. This is contrary to the findings of other studies where NAFLD (diagnosed by ultrasound) was associated with incident CKD in a community-based cohort of apparently healthy Korean men [11] independently of obesity, hypertension, and other features of metabolic syndrome. Of note, we found no gender differences in NAFLD's relationship to CKD (data not shown).

We found no association between NAFLD and CKD in individuals with diabetes in unadjusted or adjusted re- gression analyses, which contrasts with previous reports of an independent association between these two diseases in people with diabetes $[14,24]$. This difference may result from a smaller sample size of diabetic individuals in this study compared to prior studies in type 2 diabetics [13]. It is also possible that other factors such as ethnic composition, age, duration of diabetes, and dietary habits of our population may have played a role in our findings.

In addition to the cross-sectional nature of this analysis, our study has several limitations. Ultrasound is relatively insensitive to the presence of smaller amounts of hepatic steatosis, cannot distinguish nonalcoholic steatohepatitis from other forms of NAFLD, and suffers from problems with interobserver variability [25], although its noninvasive nature makes it ideal for such large epidemiological studies. In addition, while a large proportion of CKD stems from disease processes associated with metabolic syndrome, some CKD is pathogenically independent of this background (e.g. glomerulonephritis or polycystic kidney disease), and this study did not distinguish between the various causes of renal impairment.

Despite these limitations, this analysis has several important strengths. First, it is the largest and most comprehensive survey to examine the association between ultrasound-diagnosed NAFLD and the presence of CKD in a nationally representative sample of US adults. Second, NHANES-III used a standardized scoring system to screen for NAFLD with ultrasound and characterize its severity. Third, the availability of extensive and complete data on a wide range of cardiorenal risk factors allowed us to provide an unbiased estimate of the relationship between NAFLD and CKD. Finally, the design of NHANESIII allows the results to be extrapolated to the entire US civilian, noninstitutionalized, adult population. 
In conclusion, our findings demonstrate a significant positive association between the presence and severity of ultrasonographic NAFLD and CKD in US adults in unadjusted analysis. However, this association was attenuated by adjusting for features of metabolic syndrome, thus supporting the hypothesis that these two diseases may be linked as a result of the shared cardiometabolic risk factors. As such, it is of great importance to implore public health efforts and strategies to curb the epidemics of obesity and metabolic syndrome. Otherwise, the prevalence of NAFLD and CKD will continue to rise.

\section{Acknowledgements}

Dr. McFann is supported in part by NIH/NCRR Colorado CTSI Grant Number UL1 RR025780. Dr. Chonchol is supported by NIDDK grant R01DK081473-01A1 and NIDDK grant R01DK078112-01A2. Dr. Jalal is supported by grants 1K23DK088833 and R03DK094484.

\section{Disclosure Statement}

We have no conflicts of interest to declare.

\section{References}

1 Bedogni G, Miglioli L, Masutti F, Tiribelli C, Marchesini G, Bellentani S: Prevalence of and risk factors for nonalcoholic fatty liver disease: the Dionysos Nutrition and Liver Study. Hepatology 2005;42:44-52.

-2 Jimba S, Nakagami T, Takahashi M, Wakamatsu T, Hirota Y, Iwamoto Y, Wasada T: Prevalence of non-alcoholic fatty liver disease and its association with impaired glucose metabolism in Japanese adults. Diabet Med 2005;22:1141-1145.

-3 Marchesini G, Bugianesi E, Forlani G, Cerrelli F, Lenzi M, Manini R, Natale S, Vanni E, Villanova N, Melchionda N, Rizzetto M: Nonalcoholic fatty liver, steatohepatitis, and the metabolic syndrome. Hepatology 2003; 37:917-923.

-4 Targher G, Bertolini L, Padovani R, Rodella S, Tessari R, Zenari L, Day C, Arcaro G: Prevalence of nonalcoholic fatty liver disease and its association with cardiovascular disease among type 2 diabetic patients. Diabetes Care 2007;30:1212-1218.

5 Mirbagheri SA, Rashidi A, Abdi S, Saedi D, Abouzari M: Liver: an alarm for the heart? Liver Int 2007;27:891-894.

-6 Fox CS, Larson MG, Leip EP, Culleton B, Wilson PW, Levy D: Predictors of new-onset kidney disease in a community-based population. JAMA 2004;291:844-850.

7 Johns BR, Pao AC, Kim SH: Metabolic syndrome, insulin resistance and kidney function in non-diabetic individuals. Nephrol Dial Transplant 2012;27:1410-1415.

$\checkmark 8$ Thomas G, Sehgal AR, Kashyap SR, Srinivas TR, Kirwan JP, Navaneethan SD: Metabolic syndrome and kidney disease: a systematic review and meta-analysis. Clin J Am Soc Nephrol 2011;6:2364-2373.
9 Dogru T, Genc H, Tapan S, Aslan F, Ercin CN, $>17$ Edmison J, McCullough AJ: Pathogenesis of Ors F, Kara M, Sertoglu E, Karslioglu Y, Bagci S, Kurt I, Sonmez A: Plasma fetuin-A is associated with endothelial dysfunction and subclinical atherosclerosis in subjects with nonalcoholic fatty liver disease. Clin Endocrinol (Oxf) 2012, E-pub ahead of print.

10 Stefan N, Kantartzis K, Haring HU: Causes and metabolic consequences of fatty liver. Endocr Rev 2008;29:939-960

11 Chang Y, Ryu S, Sung E, Woo HY, Oh E, Cha K, Jung E, Kim WS: Nonalcoholic fatty liver disease predicts chronic kidney disease in nonhypertensive and nondiabetic Korean men. Metabolism 2008;57:569-576.

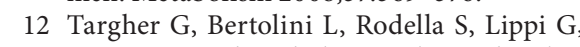
Zoppini G, Chonchol M: Relationship between kidney function and liver histology in subjects with nonalcoholic steatohepatitis. Clin J Am Soc Nephrol 2010;5:2166-2171.

13 Targher G, Bertolini L, Rodella S, Zoppini G, Lippi G, Day C, Muggeo M: Non-alcoholic fatty liver disease is independently associated with an increased prevalence of chronic kidney disease and proliferative/laser-treated retinopathy in type 2 diabetic patients. Diabetologia 2008;51:444-450.

14 Targher G, Chonchol M, Bertolini L, Rodella S, Zenari L, Lippi G, Franchini M, Zoppini G, Muggeo M: Increased risk of CKD among type 2 diabetics with nonalcoholic fatty liver disease. J Am Soc Nephrol 2008;19:15641570.

15 Hamaguchi M, Kojima T, Itoh Y, Harano Y, Fujii K, Nakajima T, Kato T, Takeda N, Okuda J, Ida K, Kawahito Y, Yoshikawa T, Okanoue T: The severity of ultrasonographic findings in nonalcoholic fatty liver disease reflects the metabolic syndrome and visceral fat accumulation. Am J Gastroenterol 2007; 102:2708-2715.

-16 Targher G, Marra F, Marchesini G: Increased risk of cardiovascular disease in non-alcoholic fatty liver disease: causal effect or epiphenomenon? Diabetologia 2008;51:19471953. non-alcoholic steatohepatitis: human data. Clin Liver Dis 2007;11:75-104, ix.

18 Albano E, Mottaran E, Occhino G, Reale E, Vidali M: Review article: role of oxidative stress in the progression of non-alcoholic steatosis. Aliment Pharmacol Ther 2005; 22(suppl 2):71-73.

19 Neuschwander-Tetri BA, Caldwell SH: Nonalcoholic steatohepatitis: summary of an AASLD single topic conference. Hepatology 2003;37:1202-1219.

20 de Alwis NM, Day CP: Non-alcoholic fatty liver disease: the mist gradually clears. J Hepatol 2008;48(suppl 1):S104-S112.

21 Targher G, Chonchol M, Zoppini G, Abaterusso $\mathrm{C}$, Bonora E: Risk of chronic kidney liver disease: is there a link? J Hepatol 2011; 54:1020-1029.

22 Carrero JJ, Park SH, Axelsson J, Lindholm B, Stenvinkel P: Cytokines, atherogenesis, and hypercatabolism in chronic kidney disease: a dreadful triad. Semin Dial 2009;22:381386.

23 Ix JH, Sharma K: Mechanisms linking obesity, chronic kidney disease, and fatty liver disease: the roles of fetuin-A, adiponectin, and AMPK. J Am Soc Nephrol 2010;21:406412 .

24 Targher G, Bertolini L, Chonchol M, Rodella S, Zoppini G, Lippi G, Zenari L, Bonora E: Non-alcoholic fatty liver disease is independently associated with an increased prevalence of chronic kidney disease and retinopathy in type 1 diabetic patients. Diabetologia 2010;53:1341-1348.

25 Schwenzer NF, Springer F, Schraml C, Stefan $\mathrm{N}$, Machann J, Schick F: Non-invasive assessment and quantification of liver steatosis by ultrasound, computed tomography and magnetic resonance. J Hepatol 2009;51:433445 . disease in patients with non-alcoholic fatty 